\title{
Theory of Doping Induced High-Spin in a Model of Polyene-Based Molecular Magnets
}

\author{
Ping Huai, Yukihiro Shimoi, and Shuji Abe \\ Nanotechnology Research Institute (NRI) and Synthetic Nano-Function Materials Project (SYNAF), \\ National Institute of Advanced Industrial Science and Technology (AIST), 1-1-1 Umezono, Tsukuba, Ibaraki 305-8568
}

\begin{abstract}
Control of intramolecular spin alignment is studied theoretically in a model of polyene-based molecular magnets in which delocalized $\pi$ electrons are coupled with localized radical spins. In a previous paper [Phys. Rev. Lett. 90 (2003) 207203], we have demonstrated that charge doping is an effective way to realize controllable high-spin in the $\pi$-conjugated molecular magnets. In this paper, we clarify the dependence of spin-alignment on the exchange interaction between the localized spin and $\pi$ electron and the electron-electron interactions. The antiferromagnetic exchange interaction plays a role different from the ferromagnetic counterpart in doped molecules. To understand complex interplay of charge and spin degrees of freedom in the doped systems, we carry out a systematic study on the phase diagram of spin alignment in the parameter space. The mechanism of the spin alignment is discussed based on the spin densities of $\pi$ electrons. The calculated results are consistent with experiments, providing a theoretical basis for the control of spin alignment.
\end{abstract}

PACS numbers: $75.50 . \mathrm{Xx}, 71.10 . \mathrm{Fd}, 75.20 . \mathrm{Hr}$

Keywords: spin alignment, charge doping, molecular magnet, exact diagonalization, Kondo-Peierls-Hubbard model

\section{INTRODUCTION}

Since the discovery of high-spin ground state in dicarbene, ${ }^{1.2}$ molecular magnetism has been extensively investigated both experimentally and theoretically for decades. $3,4,5,6$ A new class of purely organic molecular magnets has received increasing attention as their intramolecular spin alignment is controllable by external stimuli such as charge doping or photoexcitation, $7,8,9,10,11,12,13,14,15,16$ as schematically shown in Fig. 1] Those molecules share a common feature in structure: two stable radicals carrying unpaired electrons coupled with a $\pi$-conjugated moiety through exchange interaction. The two radicals are barely coupled to each other directly, but interact indirectly via $\pi$

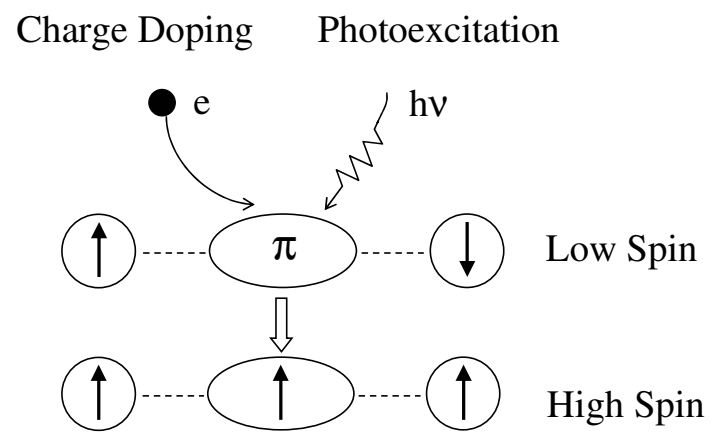

FIG. 1: Schematic picture of control of intramolecular spin alignment by charge doping or photoexcitation. can be controlled by charge doping in thianthrene bis(nitronyl nitroxide) ${ }^{7}$ The molecule is spin singlet $(S=0)$ in neutral state with very weak antiferromagnetic exchange coupling $(-1.4 \mathrm{~K})$ between the nitronyl nitroxide groups. Upon one-electron oxidation, i.e. onehole doping, spin quartet $(S=3 / 2)$ ESR signals are detected in the temperature range of $5-150 \mathrm{~K}$, indicating a low-spin to high-spin transition induced by doping. Optically controllable spin alignment has been demonstrated by Teki et al. in a molecule composed of dangling iminonitroxide radicals and $\pi$-conjugated moiety of diphenylanthracene ${ }^{10.11}$ The ground state of the molecule is determined to be a low-spin state, corresponding to weak antiferromagnetic coupling $(-5.8 \mathrm{~K})$ between the radical spins. After the molecule is excited in low temperature $(20-40 \mathrm{~K})$ by short pulses of laser, spin quintet $(S=2)$ species are observed in $10 \mu \mathrm{s}$ by using the time-resolved ESR spectroscopy. This metastable spin quintet results from photoinduced parallel spin alignment among the two stable radicals and a photogenerated triplet $\pi$ radical. Since these molecular magnets are purely organic, there is a great advantage of various chemical modifications onto the $\pi$-conjugated moiety. It might allow such molecular magnets to be building blocks of photoinduced bulk magnets similar to Prussian-blue analogs 17.18

Many theoretical works have been carried out to propose and understand the mechanism of spin alignment in molecular magnets 19.20.21.22.23.24.25.26.27.28.29.30. By a weak electron-correlation approach, Mataga predicted that ferromagnetic spin alignment appears in several hypothetical hydrocarbons with $\pi$-conjugated electron systems due to the topological nature of molecular orbitals 19 Ovchinnikov accounted for the net spins of planer alternate hydrocarbons by a strong electroncorrelation approach ${ }^{20}$ These two approaches were later generalized to rigorous theorems in the Hubbard model 
by Lieb $\stackrel{31}{3}$ Thus the dominant mechanism of intramolecular spin alignment in the ground state of $\pi$-conjugated molecules has been well established by those works: The alignment is governed by the topological rule based on the dynamical antiferromagnetic spin polarization effect of $\pi$ electrons with on-site Coulomb repulsion.

The topological rule can be applied only to the halffilled ground states, but spin alignment in doped or excited molecules remains unsolved for general cases. In the strong-correlation limit with an infinite value of onsite Coulomb repulsion in the Hubbard model, Nagaoka proved rigorously that the ferromagnetic ordering is favorable in the case of single-electron addition to or removal from half-filling 32 Most discussions on the spinalignment control so far have been given on the basis of individual molecular orbitals $\frac{8.11 .13}{2}$ For high-spin states induced by charge doping, Sakurai et al. interpreted parallel alignment of two radical spins in terms of the electronic structures calculated by the unrestricted HartreeFock theory, 8 A mechanism of photoexcited high-spin states was discussed by Teki et al. using ab initio molecular orbital calculations based on the density functional theory 11.13

Approaches by model Hamiltonians have been widely used to study the neutral ground state of quasi-onedimensional (1d) organic ferromagnets. Nasu ${ }^{22}$ studied spin alignment in an idealized poly(m-diphenylcarbene) using a periodic Kondo-Hubbard model. Fang et al ${ }^{25}$ and Chen et al ${ }^{29}$ studied a periodic Kondo Su-SchriefferHegger(SSH) mode ${ }^{33}$ taking into account the strong electron-phonon interaction as well as the electronelectron correlation. In the previous papers ${ }^{34.35}$, we introduced a Kondo-Peierls-Hubbard model to study spin alignment of $\pi$-conjugated molecular magnets. It has been demonstrated that a spin singlet to quartet transition can be induced by electronic doping into the $\pi$ moiety, which is ferromagnetically coupled with localized spins. The intramolecular spin alignment depends sensitively on the topological structure of the molecular system. The doped high-spin state can be realized in appropriate molecular structures. The topological effect in the doped case is very different from that in the half-filled case.

The aim of the present paper is to clarify the dependence of spin-alignment on spin exchange and electronelectron interactions, thereby giving an integral picture of spin-alignment control in polyene-based molecular magnets. The paper is organized as follows: In Sec. III we introduce the model and the calculation method. The calculated results are discussed in Sec. III We first study the spin alignment and the lattice deformation pattern with antiferromagnetic coupling as well as the ferromagnetic one in Sec. IIIA The dependence of spin alignment on the molecular structure is discussed in Sec. IIIB To study the complex interaction between charge and spin in doped quantum systems, we discuss the dependence of spin alignment on parameters $J$ and $U$ in Sec. IIIC All the results are summarized in Sec. IV]

\section{KONDO-PEIERLS-HUBBARD MODEL}

In this paper, we use the same Kondo-Peierls-Hubbard model as in our previous papers ${ }^{34.35}$ : a Peierls-Hubbard model on a $N$-site linear chain interacting with two localized quantum spins in the form of the Kondo coupling. The open-boundary linear chain corresponds to polyene, and its $\pi$ electrons are subject to electron-lattice interactions in the form of the $\mathrm{SSH}$ coupling 33 The localized spins correspond to the unpaired electrons of stable radical groups attached to the main chain, as realized in some substituted polyacetylenes ${ }^{36,37}$ The polyene-based molecular magnets described by this model is currently hypothetical but there is a possibility to synthesize them in future.

Thus, our model is presented in the Hamiltonian,

$$
\begin{aligned}
H= & -\sum_{i, s} t_{i}\left(C_{i, s}^{\dagger} C_{i+1, s}+\text { h.c. }\right) \\
& +U \sum_{i} n_{i, \uparrow} n_{i, \downarrow}+\frac{K}{2} \sum_{i}\left(q_{i}-q_{i+1}\right)^{2} \\
& -J\left(\mathbf{S}_{i 1} \cdot \mathbf{S}_{\mathrm{T} 1}+\mathbf{S}_{i 2} \cdot \mathbf{S}_{\mathrm{T} 2}\right) \\
t_{i} \equiv & t_{0}+\alpha\left(q_{i}-q_{i+1}\right) \\
\mathbf{S}_{i}= & \frac{1}{2} C_{i}^{\dagger} \sigma C_{i} .
\end{aligned}
$$

In Eq. (1), $C_{i, s}^{\dagger}\left(C_{i, s}\right)$ creates (annihilates) an electron with spin $s(=\uparrow$ or $\downarrow)$ on site $i$. The transfer integral $t_{i}$ is introduced for the nearest-neighbor sites, and is composed of a constant magnitude $t_{0}$, and a variable one that depends on the displacement $q_{i}$ of site $i$ with the SSH coupling constant $\alpha$. On-site Coulomb repulsion energy is given by $U$, and $n_{i, s} \equiv C_{i, s}^{\dagger} C_{i, s}$. The elastic constant of the lattice is $K$, while the kinetic energy of lattice is neglected. The last term of Eq. (1) is the exchange coupling of two localized $1 / 2$ spins $\left(\mathbf{S}_{\mathrm{T} 1}\right.$ and $\left.\mathbf{S}_{\mathrm{T} 2}\right)$ to the spins of $\pi$ electrons at site $i 1$ and $i 2$, respectively. $C_{i}^{\dagger}$ is defined as $\left(C_{i, \uparrow}^{\dagger}, C_{i, \downarrow}^{\dagger}\right)$ and $\sigma \equiv\left(\sigma_{x}, \sigma_{y}, \sigma_{z}\right)$ are the Pauli matrices. We define a dimensionless coupling constant $\lambda \equiv \alpha^{2} /\left(t_{0} K\right)$ and dimensionless bondlengths $\Delta_{i} \equiv \alpha\left(q_{i}-q_{i+1}\right) / t_{0}$.

We exactly diagonalize the electronic and spin part of the Hamiltonian by the Lanczos algorithm. Therefore the obtained wavefunctions take account of all the correlation effects. The lattice deformation is treated classically and optimized by means of the Hellmann-Feynman force equilibrium condition at zero temperature,

$$
\Delta_{i}=\lambda\left\langle\sum_{s}\left(C_{i, s}^{\dagger} C_{i+1, s}+\text { h.c. }\right)\right\rangle,
$$

where $\langle\cdots\rangle$ denotes the ground state expectation value. We perform these two procedures iteratively until the electronic state and lattice deformation converge. Since the model has the electron-hole symmetry, electron doping gives the same results as hole doping. Only the results for hole doping will be discussed in the paper. 
First let us analyze the case of strong-correlation limit $U / t_{0} \rightarrow \infty$. The half-filled Hubbard model is mapped onto the Heisenberg model with effective exchange interactions $J_{i}^{\text {eff }}=-4 t_{i}^{2} / U(<0)$, so that the electronic and spin parts of the present Hamiltonian is reduced to

$$
\begin{aligned}
H_{e f f}= & -\sum_{i} J_{i}^{\mathrm{eff}}\left(\mathbf{S}_{i} \mathbf{S}_{i+1}-1 / 4\right) \\
& -J\left(\mathbf{S}_{i 1} \cdot \mathbf{S}_{\mathrm{T} 1}+\mathbf{S}_{i 2} \cdot \mathbf{S}_{\mathrm{T} 2}\right) .
\end{aligned}
$$

The spin alignment predicted by the topological rule can be deduced from such a spin Hamiltonian in an intuitive way, if quantum fluctuations are neglected. Because of antiferromagnetic correlation between the neighboring $\pi$ spins, the alignment between $\mathbf{S}_{T 1}$ and $\mathbf{S}_{T 2}$ can be classified in terms of the parity of $i 2-i 1$ : antiparallel if $i 2-i 1$ is odd, but parallel if $i 2-i 1$ is even, no matter whether the exchange coupling $J$ is ferromagnetic or antiferromagnetic. For $J<0$, this intuitive picture is consistent with rigorous theorems ${ }^{20.39}$ On the other hand, single hole doping induces Nagaoka ferromagnetic ordering in the limit of strong correlation ${ }^{32}$ Therefore the two localized spins favor parallel alignment. If $U / t$ is finite, no simple rule exists for the doped $\pi$ electron systems, and numerical methods are more effective way to study doped states.

The model is investigated with the following parameters in the unit of $t_{0}$ (on the order of $\mathrm{eV}$ ): $U=2.5$, $|J|=0.2$ while $\lambda$ is allowed to vary from 0 to 0.5 . In the previous paper ${ }^{34}$, we assumed the exchange interaction much stronger $(|J|=1)$ than this value, and discussed mostly the case of ferromagnetic coupling. Here, weaker exchange interaction is applied to the model because the reduced value gives more realistic spin gap. Furthermore, $J$ will be discussed for both ferromagnetic (F) $J>0$ and antiferromagnetic (AF) $J<0$, although $J$ has been suggested to be positive in the thianthrene-based molecular magnets 8 .

\section{RESULTS AND DISCUSSION}

\section{A. Spin correlations and lattice deformations}

To investigate spin alignment with electron-lattice interaction, we first study a 10-site chain with $\lambda=0.25$ (a typical value for polyacetylene ${ }^{33.38}$ ). The two localized spin are coupled to electron spin at both ends of the chain, i.e. $i 1=1$ and $i 2=10$. The spin correlations are plotted in Fig. 2 for both the half-filled $\left(N_{e}=10\right)$ and the single hole doped $\left(N_{e}=9\right)$ cases.

The correlations between $\mathbf{S}_{\mathrm{T} 1}$ and the other spins are shown in Fig. 2(a) for the case of F coupling $(J>0)$. The correlation between $\mathbf{S}_{\mathrm{T} 1}$ and $\mathbf{S}_{\mathrm{T} 2}$ is antiferromagnetic for the half-filled case, resulting in spin singlet $S=0$. This antiparallel spin alignment can be understood based on the topological rule for the half-filled ground state. The spin correlation $\left(\left\langle\mathbf{S}_{\mathrm{T} 1} \cdot \mathbf{S}_{\mathrm{T} 2}\right\rangle=-0.745\right)$ is near to $-3 / 4$ of a singlet pair of the localized spins.

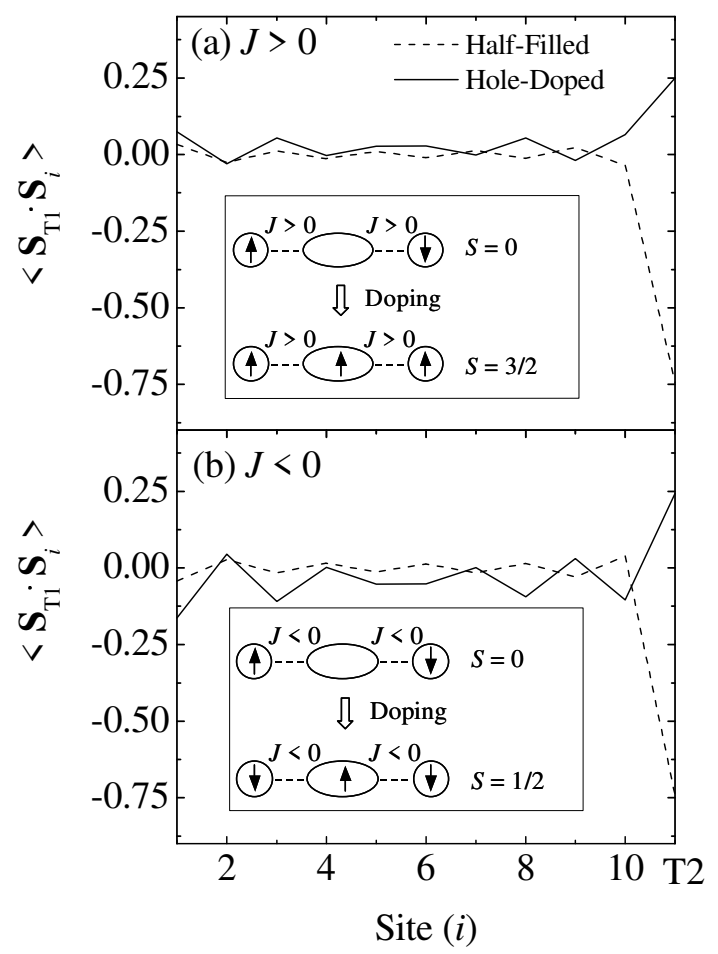

FIG. 2: Spin correlations $\left\langle\mathbf{S}_{\mathrm{T} 1} \cdot \mathbf{S}_{i}\right\rangle$ for the half-filled $\left(N_{e}=N\right.$; dashed line) and the single hole doped cases $\left(N_{e}=N-1\right.$; solid line) with $|J|=0.2, U=2.5, \lambda=0.25$, and $N=10$. (a) F coupling $(J>0)$ and (b) AF coupling $(J<0)$. The inset in each figure shows schematically the change of spin alignment by hole doping.

Positive correlation appears between the localized spins when the $\pi$ electron moiety is doped by a hole. Similar spin alignment has been reported in the case of the stronger coupling 34 . In the present case, this spin correlation $\left(\left\langle\mathbf{S}_{\mathrm{T} 1} \cdot \mathbf{S}_{\mathrm{T} 2}\right\rangle=0.249\right)$ is near to $1 / 4$ of a triplet pair of the localized spins. The spin correlation pattern in the right half of the chain $(i \geq 6)$ is also reversed by the doping. Furthermore, the doped system turns out to be a spin quartet $(S=3 / 2)$. The inset in Fig. 2(a) illustrates the alteration of spin alignment from the spin singlet to the spin quartet by such hole doping. This kind of low-spin to high-spin transition corresponds to the observation in the aforementioned thianthrene derivative ${ }^{7}$

Figure 2(b) shows the spin correlation in the case of AF coupling $(J<0)$. Antiferromagnetic correlation between $\mathbf{S}_{\mathrm{T} 1}$ and $\mathbf{S}_{\mathrm{T} 2}(=-0.742)$ is found in the halffilled case with the total spin $S=0$, which is also expected from the topological rule. However, hole doping induces a spin doublet $S=1 / 2$, while the correlation $\left(\left\langle\mathbf{S}_{\mathrm{T} 1} \cdot \mathbf{S}_{\mathrm{T} 2}\right\rangle=0.243\right)$ is ferromagnetic similar to the case of $\mathrm{F}$ coupling. The antiparallel alignment between the hole spin and the localized spins is responsible for the spin doublet as schematically shown in the inset of Fig. [2(b).

The lattice deformation patterns is plotted in Fig. 3 


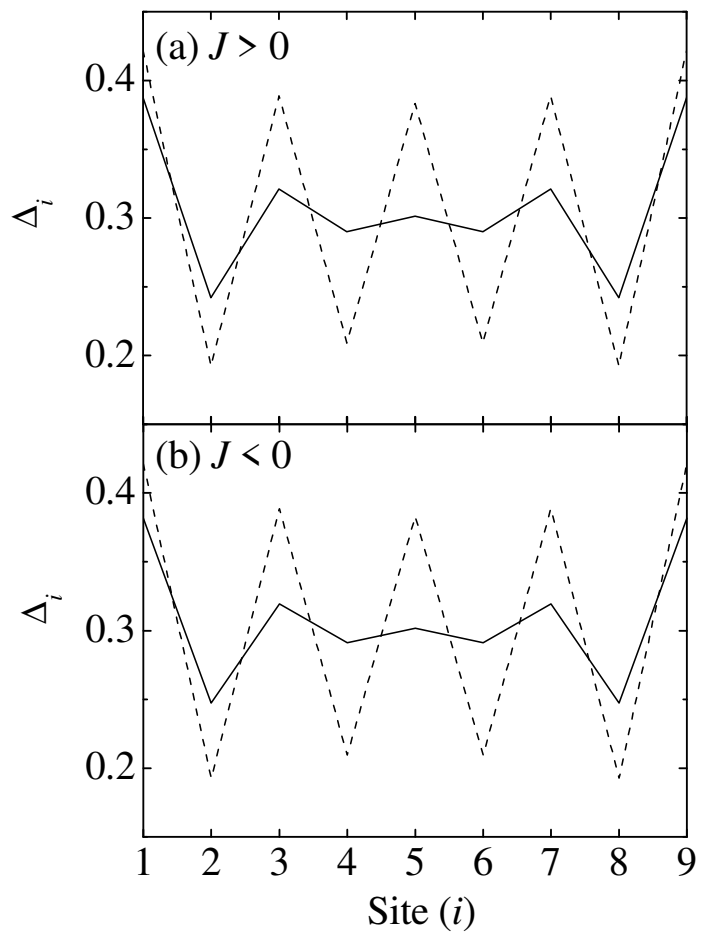

FIG. 3: Lattice deformations $\Delta_{i}$ for the half-filled $\left(N_{e}=N\right.$; dashed line) and the single hole doped cases $\left(N_{e}=N-1\right.$; solid line) with $|J|=0.2, U=2.5, \lambda=0.25$, and $N=10$. (a) F coupling $(J>0)$ and (b) AF coupling $(J<0)$.

for both $\mathrm{F}$ and $\mathrm{AF}$ couplings. Quite similar profiles are obtained in both cases: half-filled case has bond alternation in the lattice, while the doped case contains very weak bond alternation especially around the middle of the lattice. The localization of the lattice relaxation is ascribed to a polaronic effect as well as a chain-end effect as shown in ref. 40.

We examine the effect of electron-lattice coupling on spin alignment by investigating spin correlation and gap energy between the ground state and the first excited state in the range of $0 \leq \lambda \leq 0.5$. The $\lambda$-dependences are plotted in Fig. 4 for F coupling. For both the halffilled and the doped cases, the spin correlation is barely affected by $\lambda$. The inset in Fig. 目 b) indicates that the lowest excitation takes place from singlet to triplet in the half-filled case, and from quartet to doublet in the doped case. In the half-filled case with $\lambda=0.25$, the spin gap $\left(5.5 \times 10^{-4} t_{0}\right.$, about a few $\left.\mathrm{K}\right)$ is comparable to the effective couplings of thianthrene bis(nitronyl nitroxide $)^{7}$. The spin gap gradually decreases as the electron-lattice coupling increases, because of the increasing tendency of local singlet-pair formation in $\pi$ electrons. The gap in the doped case is an order of magnitude larger than that in the half-filled case, and decreases as $\lambda$ increases. Spin polarization is suppressed by strong bond alteration as electron-lattice coupling increases. The spin gaps of very weakly coupled systems tend to decrease with reduction

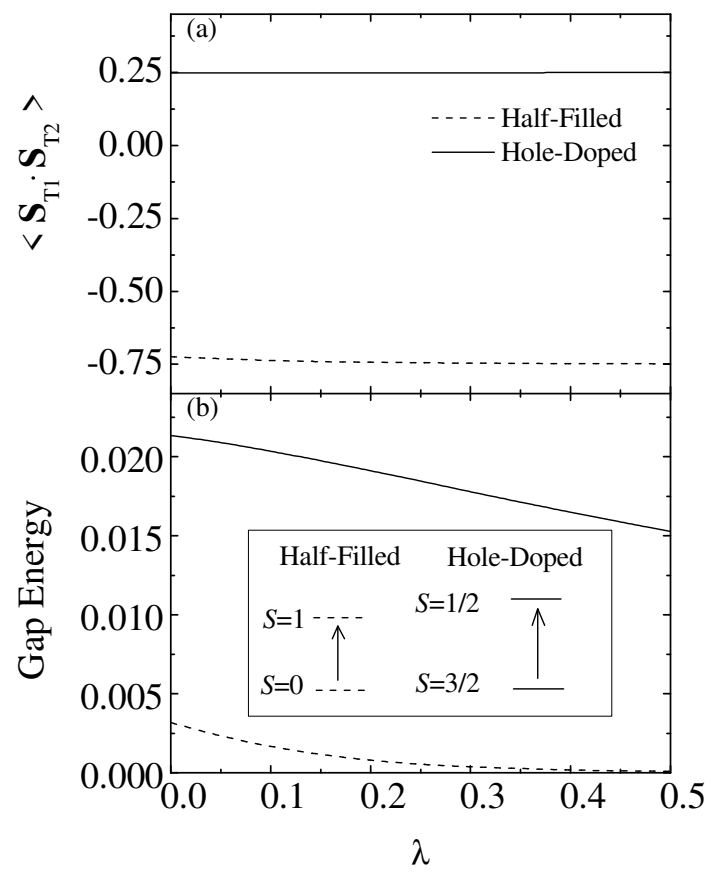

FIG. 4: (a) Spin correlations $\left\langle\mathbf{S}_{\mathrm{T} 1} \cdot \mathbf{S}_{\mathrm{T} 2}\right\rangle$ and (b) gap energies as functions of $\lambda$ for the half-filled $\left(N_{e}=N\right.$; dashed line) and the doped $\left(N_{e}=N-1\right.$; solid line) cases with $J=0.2$, $U=2.5, \lambda=0.25$, and $N=10$. The inset in (b): the total spins of the ground and the first excited states.

of spin densities on the coupling sites. This $\lambda$-dependence of the spin gap is different from that reported in our previous paper with a larger $J^{34}$. In the case of strong exchange interaction, the localized spins are firmly bound to the $\pi$-spins, and are hardly set free by low-energy excitations. Thus the spin gap would be mainly governed by excitations within the $\pi$-moiety. These $\pi$-excitation energies are raised by the electron-lattice coupling, resulting in the increase of the spin gap with $\lambda$ in the stronger $J$ case.

For the AF coupling, the spin correlation and the gap energy are plotted as functions of $\lambda$ in Fig. 5 We see tendencies similar to the case of the F coupling, even though the manner of spin alignment in the doped state depends on the sign of the coupling, as already seen in the insets of Fig. 22 In the half-filled case with $\lambda=$ 0.25 , the spin gap $\left(9.5 \times 10^{-4} t_{0}\right)$ is larger than that of the $\mathrm{F}$ coupling but is still fairly small against thermal fluctuations at room temperature. The suppression of electron-lattice coupling is desirable in molecular design with respect to the thermal stability.

\section{B. Effects of molecular structure}

Geometrical structures are very important to $\pi$ conjugated molecular magnets. The influence on the doped state is different from that on the neutral one, as 


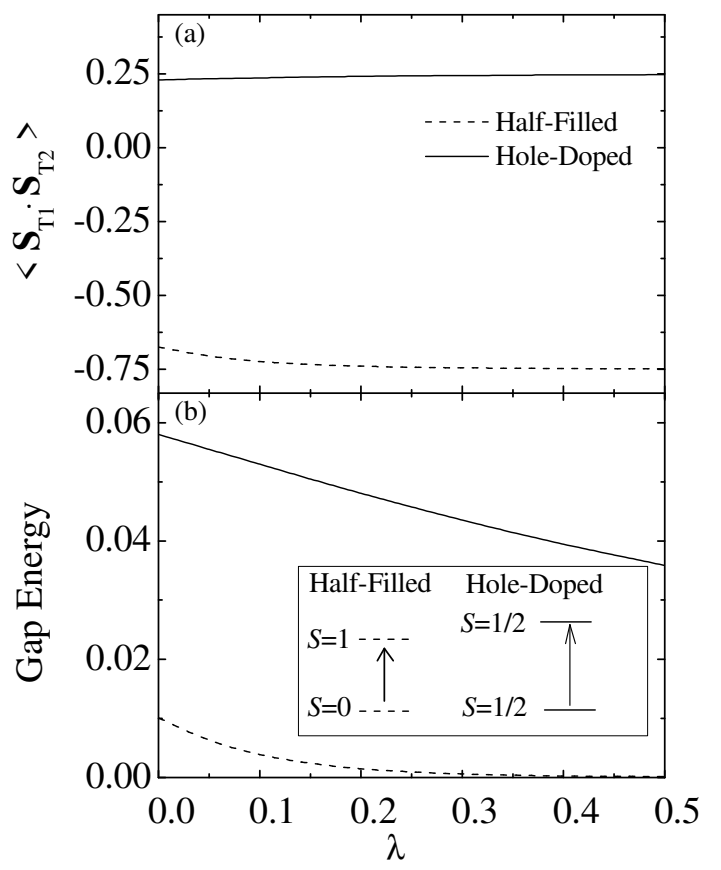

FIG. 5: (a) Spin correlations $\left\langle\mathbf{S}_{\mathrm{T} 1} \cdot \mathbf{S}_{\mathrm{T} 2}\right\rangle$ and (b) gap energie: as functions of $\lambda$ for the half-filled $\left(N_{e}=N\right.$; dashed line and the doped $\left(N_{e}=N-1\right.$; solid line) cases with $J=-0.2$ $U=2.5, \lambda=0.25$, and $N=10$. The inset in (b): the tota spins of the ground and the first excited states.

pointed out in the previous paper ${ }^{34}$. It has been observec in the topological isomers of thianthrene bis(nitronyl ni troxide), that spin state depends on the positions of the radicals in the neutral case but has no positiondependence in the doped $\operatorname{case}^{7}$. In this paper, we examine two geometrical factors: the length of the chain $N$ and the positions of the localized spins.

First we examine the $N$-dependence of spin alignment. Figure [6] shows the correlations $\left\langle\mathbf{S}_{\mathrm{T} 1} \cdot \mathbf{S}_{\mathrm{T} 2}\right\rangle$ between the localized spins, the total spins, and the gap energies as functions of $N$ in the case of the $\mathrm{F}$ coupling. To focus on the effect of the length, we maintain the localized spins at both ends of the chain. In the half-filled case, the topological rule governs the spin correlation and total spin perfectly. For even number of $N$, spin state is singlet $(S=0)$ with antiparallel alignment between $\mathbf{S}_{\mathrm{T} 1}$ and $\mathbf{S}_{\mathrm{T} 2}$, while for the odd number case total spin is quartet $(S=3 / 2)$ with parallel alignment.

Similar alternating behavior is also seen in the holedoped systems, although the topological rule is generally only applicable for the ground state of the half-filled system. The hole doping changes the total spin and spin correlation $\left\langle\mathbf{S}_{\mathrm{T} 1} \cdot \mathbf{S}_{\mathrm{T} 2}\right\rangle$ as follows: $S=0 \rightarrow S=3 / 2$ with $\left\langle\mathbf{S}_{\mathrm{T} 1} \cdot \mathbf{S}_{\mathrm{T} 2}\right\rangle>0 \rightarrow\left\langle\mathbf{S}_{\mathrm{T} 1} \cdot \mathbf{S}_{\mathrm{T} 2}\right\rangle<0$, if $N$ is even; $S=3 / 2 \rightarrow S=0$ with $\left\langle\mathbf{S}_{\mathrm{T} 1} \cdot \mathbf{S}_{\mathrm{T} 2}\right\rangle\left\langle 0 \rightarrow\left\langle\mathbf{S}_{\mathrm{T} 1} \cdot \mathbf{S}_{\mathrm{T} 2}\right\rangle>0\right.$, if $N$ is odd. It is noted that the spin correlation does not decay with increasing the chain length both in the doped and half-filled cases, while the spin gap decreases gradu-

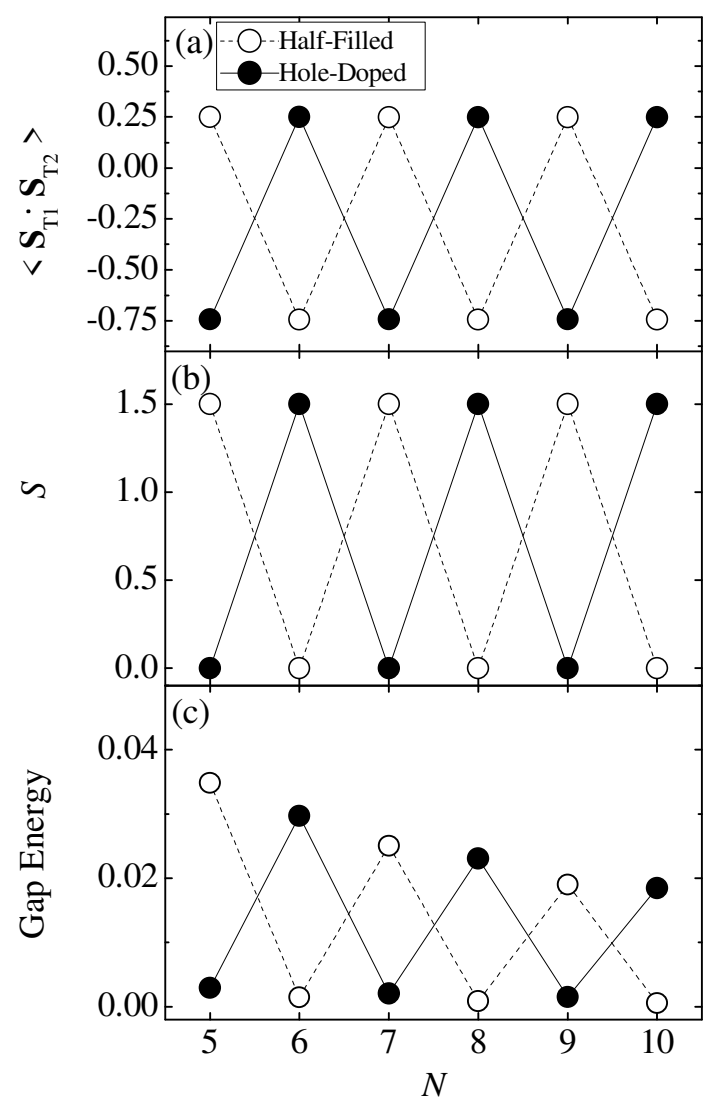

FIG. 6: (a) Spin correlations $\left\langle\mathbf{S}_{\mathrm{T} 1} \cdot \mathbf{S}_{\mathrm{T} 2}\right\rangle$, (b) total spins, and (c) gap energies as functions of $N$ for the half-filled $\left(N_{e}=N\right.$; open circle) and the doped $\left(N_{e}=N-1\right.$; filled circle) cases with $J=0.2, U=2.5$, and $\lambda=0.25$.

ally as shown in Fig. 6(c). In general, one-dimensional electron systems have no long-range charge (spin) order as a result of quantum fluctuations. The correlation between a pair of electrons decays in a manner of power law with increasing distance. Decreasing electron correlation would weaken the interaction between the localized spins in our model, and reduce the spin gap as the chain becomes longer.

We turn to the case of the AF coupling shown in Fig. 7 In the half-filled system, the alignment of $\mathbf{S}_{\mathrm{T} 1}$ and $\mathbf{S}_{\mathrm{T} 2}$ is determined by the parity of $N$ in the same manner of the F coupling: antiparallel if $N$ is even, parallel if $N$ is odd. In contrast to the $\mathrm{F}$ coupling, the total spin is singlet $(S=0)$ for even $N$, and doublet $(S=1 / 2)$ for odd $N$, as shown in Fig. 7 (b). The single hole doping flips the localized spin from antiparallel to parallel with $S=0 \rightarrow S=1 / 2$ if $N$ is even, and from parallel to antiparallel with $S=1 / 2 \rightarrow S=0$ if $N$ is odd. The $N$-dependence of the spin correlation and of the spin gap is similar to the case of the F coupling.

Next we proceed to the other geometrical factor, considering the localized spins attached to inner sites of the chain. We pick up symmetric positions of the localized 


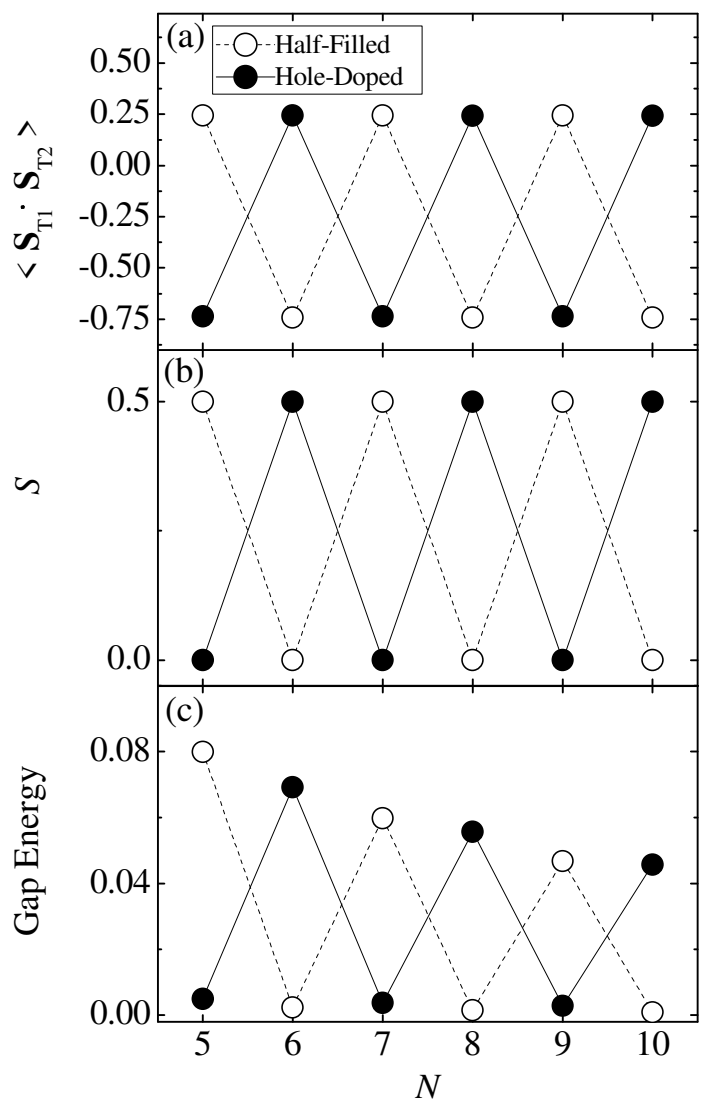

FIG. 7: (a) Spin correlations $\left\langle\mathbf{S}_{\mathrm{T} 1} \cdot \mathbf{S}_{\mathrm{T} 2}\right\rangle$, (b) total spins, and (c) gap energies as functions of $N$ for the half-filled $\left(N_{e}=N\right.$; open circle) and the doped $\left(N_{e}=N-1\right.$; filled circle) cases with $J=-0.2, U=2.5$, and $\lambda=0.25$.

spins as $(i 1, i 2)=(1, N),(2, N-1), \cdots$, with $N=10$. In Fig. 8 the spin correlations $\left\langle\mathbf{S}_{\mathrm{T} 1} \cdot \mathbf{S}_{\mathrm{T} 2}\right\rangle,\left\langle\mathbf{S}_{\mathrm{T} 1} \cdot \mathbf{S}_{\mathrm{e}}\right\rangle$ $\left(\mathbf{S}_{\mathrm{e}} \equiv \sum_{i=1}^{N} \mathbf{S}_{i}\right)$, and the total spin are plotted as functions of $i 1$ for the $\mathrm{F}$ coupling. In the half-filled case, these values are almost independent of $i 1$ : the spin alignment is antiparallel with $S=0$, as expected by the topological rule, since $i 2-i 1$ is odd. The correlation between $\mathbf{S}_{\mathrm{T} 1}$ and $\mathbf{S}_{\mathrm{e}}$ is negligible, because $\left\langle\mathbf{S}_{\mathrm{e}}\right\rangle$ is almost zero in the half-filled electron systems.

For the doped case, the most interesting feature is that the relative alignment among the two localized spins and the hole spin depends on $i 1$ and $i 2$. In Fig. 8 (a), the localized spins exhibit positive correlation $\left\langle\mathbf{S}_{\mathrm{T} 1} \cdot \mathbf{S}_{\mathrm{T} 2}\right\rangle$ except for the position $(i 1, i 2)=(4,7)$. Furthermore, these positively correlated spins can be classified into two categories in terms of $\left\langle\mathbf{S}_{\mathrm{T} 1} \cdot \mathbf{S}_{\mathrm{e}}\right\rangle$, namely the alignment between the localized spin and the hole spin. They are parallel in the case of $i 1=1,3$, and 5 but antiparallel in the case of $i 1=2$ (Fig. \& (b)). Correspondingly the doped systems are spin quartet $(S=3 / 2)$ for the former three structures but spin doublet $(S=1 / 2)$ for the last one (Fig. $8(\mathrm{c}))$. For $(i 1, i 2)=(4,7)$, the localized spins are in antiparallel alignment leading to spin doublet

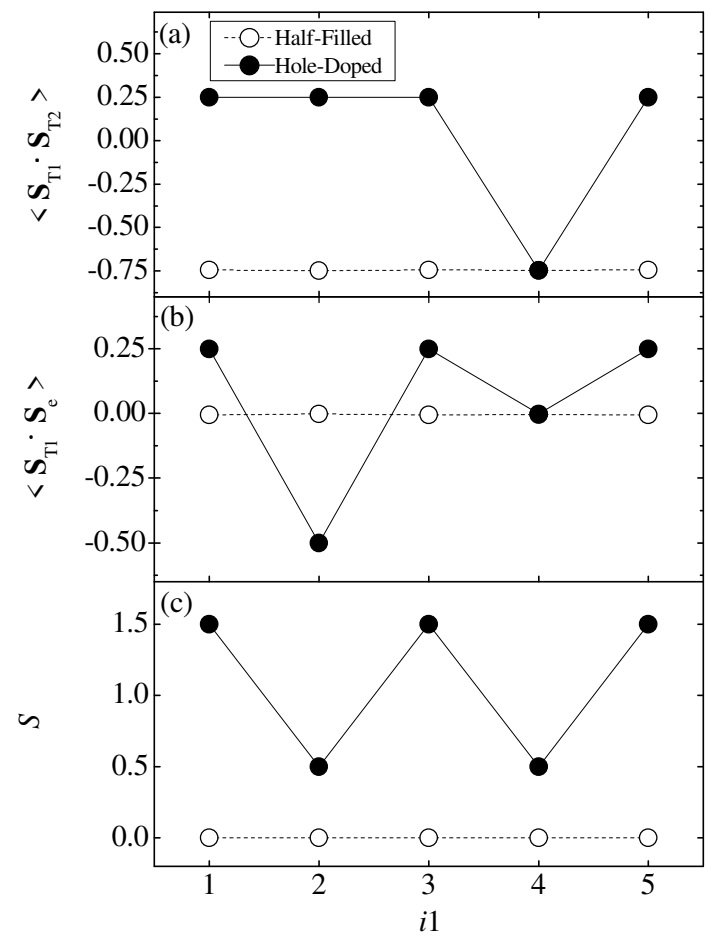

FIG. 8: (a) Spin correlations $\left\langle\mathbf{S}_{\mathrm{T} 1} \cdot \mathbf{S}_{\mathrm{T} 2}\right\rangle$, (b) $\left\langle\mathbf{S}_{\mathrm{T} 1} \cdot \mathbf{S}_{\mathrm{e}}\right\rangle$, and (c) total spins as functions of $i 1$, with $i 2=N+1-i 1$, for the half-filled $\left(N_{e}=N\right.$; open circle) and the doped $\left(N_{e}=N-1\right.$; filled circle) cases with $J=0.2, U=2.5, \lambda=0.25$, and $N=10$.

$(S=1 / 2)$.

Figure 9 shows results for the AF coupling as the localized spins are attached to inner sites of the chain. Just like the $\mathrm{F}$ coupling, the half-filled systems do not exhibit the $i 1$-dependence: they are all spin singlet $(S=0)$ with antiparallel alignment between the localized spins. In the doped systems, the localized spins show parallel alignment in all the cases as shown in Fig. 9(a). On the other hand, they show antiparallel alignment with the hole spin except for $i 1=2$ (Fig. 9(b)). This difference in spin alignment results in the different total spin: Spin doublet $(S=1 / 2)$ for $i 1=1,3,4$, and 5 and spin quartet $(S=3 / 2)$ for $i 1=2$ (Fig. 9(c)).

The gap energies of the doped systems are presented in Fig. 10] The gap is relatively large at $i 1=1$ and 3 for both $J>0$ and $J<0$, where the high-spin states appear in the case of $J>0$. All the results above imply that molecular systems with particular structures are suitable for the purpose of spin-alignment control since they exhibit doping-induced high-spin and have large spin gaps.

\section{Dependence on $J$ and $U$}

It is a key issue in the present study to understand the complex interplay of spin and charge degrees of freedom in doped quantum systems. We have demonstrated 


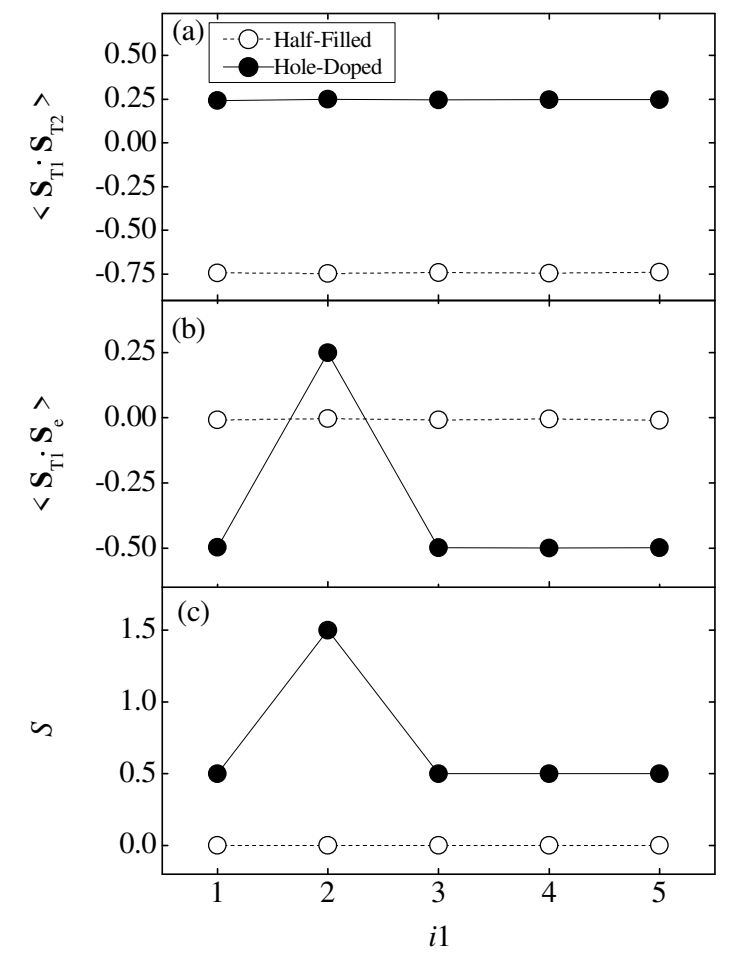

FIG. 9: (a) Spin correlations $\left\langle\mathbf{S}_{\mathrm{T} 1} \cdot \mathbf{S}_{\mathrm{T} 2}\right\rangle$ and (b) $\left\langle\mathbf{S}_{\mathrm{T} 1} \cdot \mathbf{S}_{\mathrm{e}}\right\rangle$, and (c) total spins as functions of $i 1$, with $i 2=N+1-i 1$, for the half-filled $\left(N_{e}=N\right.$; open circle) and the doped $\left(N_{e}=N-1\right.$; filled circle) cases with $J=-0.2, U=2.5, \lambda=0.25$, and $N=10$.

the spin-alignment control by hole-doping using fixed parameters of the on-site Coulomb repulsion $U$ and the exchange interaction $J$. However, a subtle balance between these interactions governs the alignment between the separated radical spins. It leads to a question: Does spin alignment change dramatically with varying the strengths of $U$ and $J$, or just depend on them quantitatively? As already shown in Fig. [10, the spin gap is sometimes very small in the doped systems, hence the order of the present lowest excited and ground states could be interchanged for another set of parameters. Furthermore, the spin alignment depends on the topological structure as shown in Sec. IIIB Thus, it is necessary to investigate the dependence on $U$ and $J$ for various molecular structures. In the following discussion, the electronlattice coupling is omitted as it plays less important role than the electron-electron interaction.

In the case of $\mathrm{F}$ coupling, the phase diagrams in the parameter space of $J$ and $U$ are shown for $i 1=2,4$, and 5 in Fig. 11] The doped systems with $i 1=1$ or 3 are always spin quartet in the calculated range of $0<J<0.5$ and $0<U<8$. As the on-site Coulomb interaction increases over a critical value about $U \sim 0.4$ for $i 1=2$, or $U \sim 2.0$ for $i 1=4$, the systems are transferred from the spin quartet to the spin doublet, and the phase boundaries are almost independent of the exchange interaction.

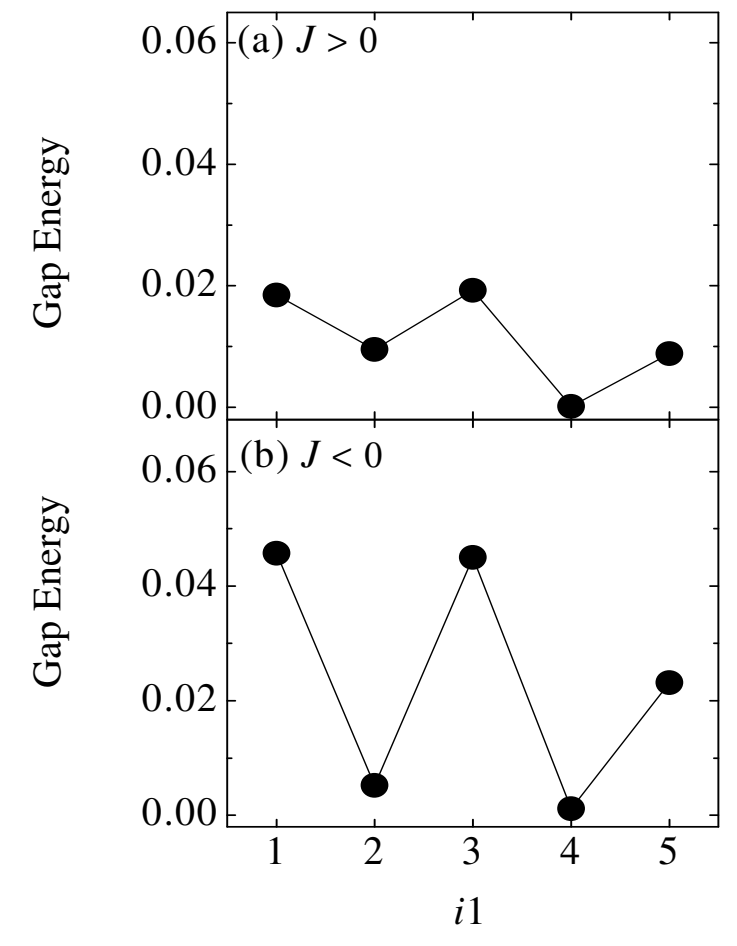

FIG. 10: Gap energies of the doped system: (a) $J>0$ and (b) $J<0$ as functions of $i 1(i 2=N+1-i 1)$, with $|J|=0.2$, $U=2.5, \lambda=0.25$, and $N=10$.

The system with $i 1=5$ has much strong dependence on $J$ and $U$. It also undergoes a transition from the spin quartet to the spin doublet, but occurs in stronger Coulomb interaction $U>4.0$. Detailed analysis reveals that the spin gap goes continuously to zero when the system approaches to the phase boundary.

Similar transitions are also found in the case of $\mathrm{AF}$ coupling. Figure 12 shows the phase diagrams for the systems with $i 1=2$ and 4 , which are transferred from spin doublet to quartet at $U \sim 0.4$ for $i 1=2$ or at $U \sim 2.0$ for $i 1=4$ in a way opposite to the case of $\mathrm{F}$ coupling. The transitions occur at similar values of $U$ in both the $\mathrm{F}$ and AF couplings. However, $J$-dependence is opposite between them. The systems with $i 1=1,3$, and 5 do not exhibit phase transitions in the calculated range of $-0.5<J<0$ and $0<U<8$.

To understand the origin of these phase transitions, we calculated the doped states in the 1d Hubbard model $\left(N=10, N_{\uparrow}=5, N_{\downarrow}=4\right)$, which is nothing but setting $J=0$ in our model. Their spin densities are plotted as functions of $\operatorname{site}(i)$ in Fig. 13(a). The spin density is positive at every site if the on-site Coulomb interaction is absent or quite weak. As the strength of $U$ increases, spin densities become negative first at $i=2$ and 9 for $U>0.4$, and then at site $i=4$ and 7 for $U>2.0$. The spin densities at the other sites remain positive irrespective of the strength of $U$. We also plot the spin correlation $\left\langle\mathbf{S}_{i 1} \cdot \mathbf{S}_{i 2}\right\rangle$ with $i 2=N+1-i 1$ in Fig. 13(b). The 


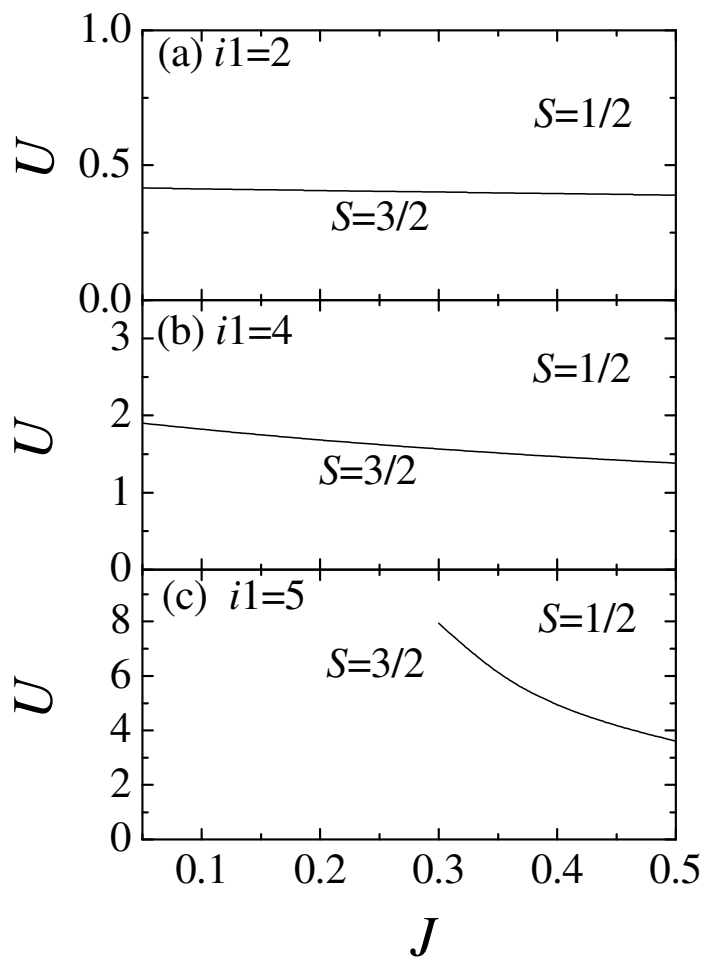

FIG. 11: Phase diagrams of the doped system $\left(N_{e}=N-1\right)$ for (a) $i 1=2$, (b) $i 1=4$, and (c) $i 1=5,(i 2=N+1-i 1)$ with $J>0, \lambda=0$, and $N=10$.

correlations are positive for $i 1=1$ and 3 , negative for $i 1=4$ and 5 , and almost zero for $i 1=2$.

Returning to our original model with the localized spins attached, we can understand the positiondependent alignment of the spins shown in Figs. 8 and 9 from the spin densities of the $\pi$ electrons shown in Fig. 133(a). In the case of F coupling, each localized spin tends to be in the parallel alignment with the $\pi$-spin at its coupling site. If the coupling site has a positive spin density, the localized spin is in the parallel alignment with the hole spin leading to a spin quartet, as illustrated in Fig. 14(a). On the other hand, the radical spin is in the antiparallel alignment with the hole spin if the coupling site has a negative spin density, resulting in a spin doublet as schematically shown in Fig. 14(b). In addition, we can also explain the critical values of $U$ in the phase diagrams of Fig. [1] in the limit of $J \rightarrow 0$ : The transition point is close to the value of $U$ where the spin density becomes negative in Fig. 13(a). The spin alignment in the case of AF coupling can also be understood in a similar way as shown in Fig. 14(c) and (d). This picture is consistent with the spin correlation $\left\langle\mathbf{S}_{i 1} \cdot \mathbf{S}_{i 2}\right\rangle$, which is positive for large $U$ at $i 1=1,2$, and 3 as shown in Fig. 13(b). As for $i 1=5$, the spin alignment for both $\mathrm{F}$ and $\mathrm{AF}$ couplings can be explained based on the spin densities, although the spin correlation $\left\langle\mathbf{S}_{\mathrm{i} 1} \cdot \mathbf{S}_{\mathrm{i} 2}\right\rangle$ is negative.

The absolute value of the spin density at $i 1=4$ is very small, and the parameter set is close to the phase

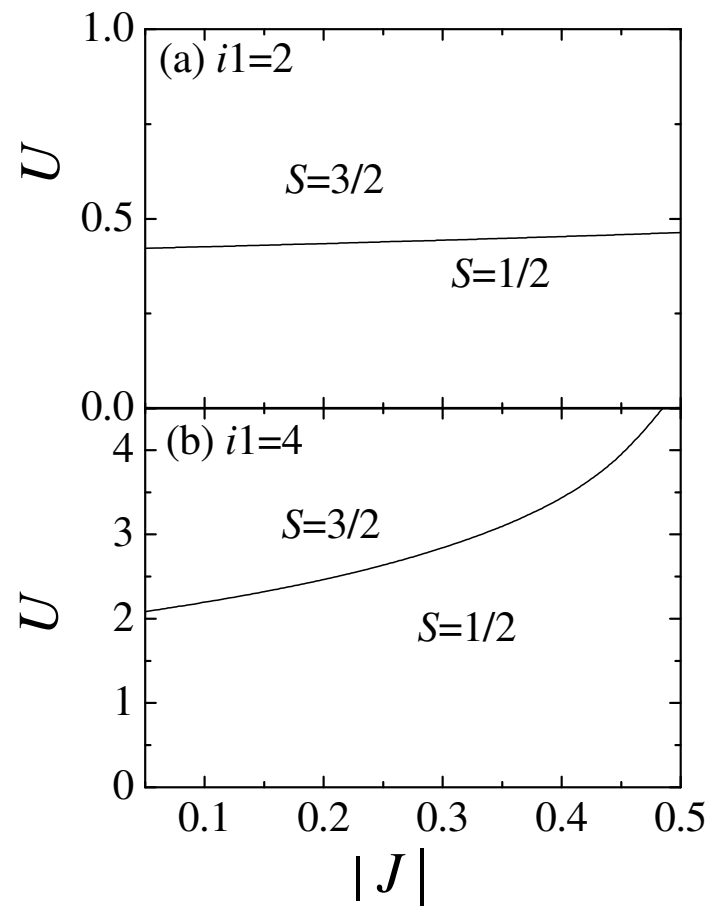

FIG. 12: Phase diagrams of doped system $\left(N_{e}=N-1\right)$ for (a) $i 1=2$ and (b) $i 1=4,(i 2=N+1-i 1)$ with $J<0, \lambda=0.0$ and $N=10$.

boundary as shown in Figs. 12(b). The sign of spin density at this site can be changed to positive by a finite electron-lattice coupling. As a result, spin doublet appears in the case of AF coupling, as schematically shown in Fig. 14(c). The spin correlation $\left\langle\mathbf{S}_{4} \cdot \mathbf{S}_{7}\right\rangle$ is negative as shown in Fig. 13(b), and takes control of the spin alignment in the case of $\mathrm{F}$ coupling. This spin alignment leads to antiferromagnetic correlation between the localized spins, and results in a spin doublet. Such situation appears also at $i 1=5$ for the case of stronger coupling in our previous paper $\stackrel{34}{ }$.

\section{SUMMARY}

In summary, the control of spin alignment by charge doping is studied in the theoretical model of polyenebased molecular magnets. By mean of the exact diagonalization technique, we study the spin alignment, the total spins and gap energies in the doped and the halffilled states.

Low-spin to high-spin transition is realized in such polyene-based molecular magnets by one-hole doping into the $\pi$-conjugated moiety. The doping-induced spin alignment depends on the molecular structure and the strengths of the interactions. Alternating behavior of spin alignment is demonstrated in the hole-doped systems with growth of the chain length. Variation of spincoupling positions leads to different alignment among the 

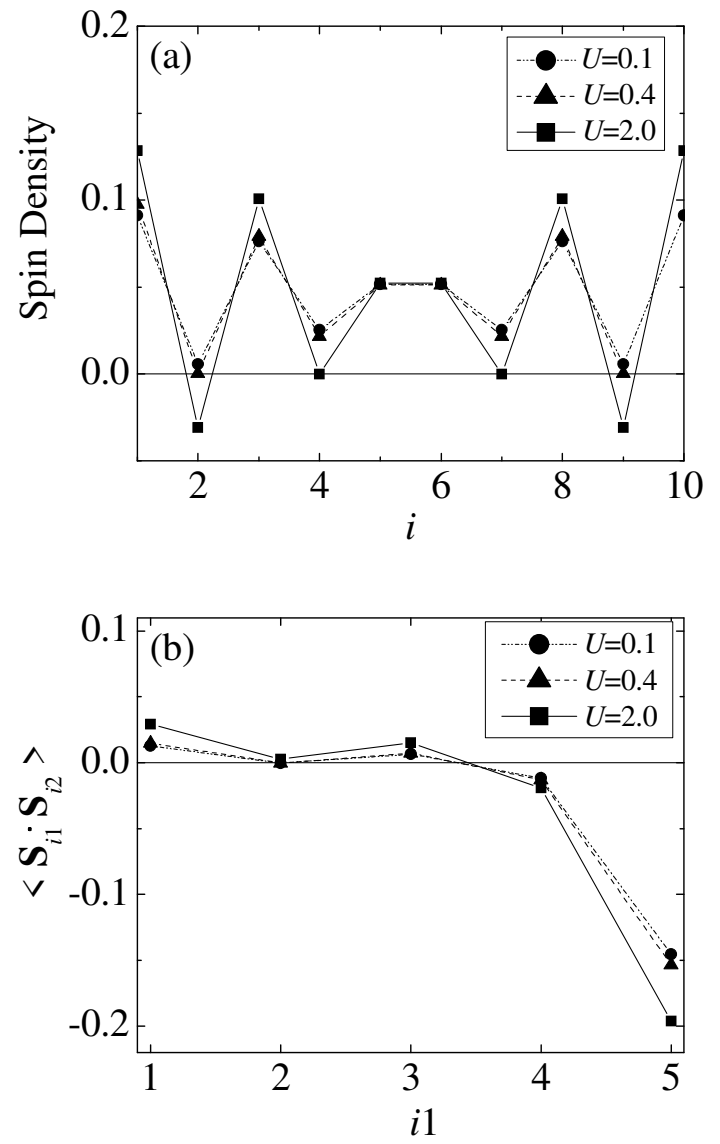

FIG. 13: (a)Spin densities $\left\langle n_{i, \uparrow}-n_{i, \downarrow}\right\rangle / 2$ as a function of $i$; (b spin correlations $\left\langle\mathbf{S}_{i 1} \cdot \mathbf{S}_{i 2}\right\rangle$ as a function of $i 1(i 2=N+1-i 1$ for the doped state in the 1d Hubbard model $\left(N=10, N_{\uparrow}=\right.$ $5, N_{\downarrow}=4$ ) with open boundary condition. The dash-do1 dash, and solid lines correspond to $U=0.1,0.4$, and $2 .($ respectively.

localized spins and the hole spin. To understand behav iors in doped system, we investigate the dependence $c$ spin alignment on $J$ and $U$ in detail. The antiferromag netic and ferromagnetic exchange interactions have dif ferent effect on doped molecules. The overall behavior c spin alignment can be understood in terms of spin density and correlation of $\pi$ electrons, in contrast to the $\pi$ topological rule for the half-filled case where antiferromagnetic correlation in $\pi$ electrons governs spin alignment.

We have shown that charge doping is an effective way to realize controllable spin alignment in molecular magnets. As an important step towards molecular designing. (a)

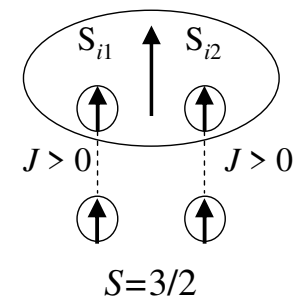

(c)

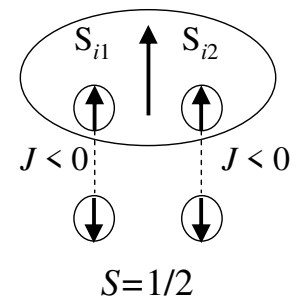

(b)

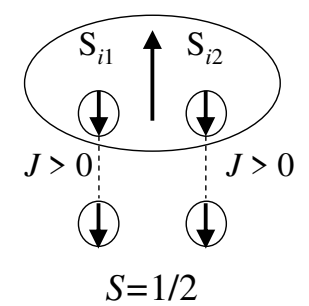

(d)

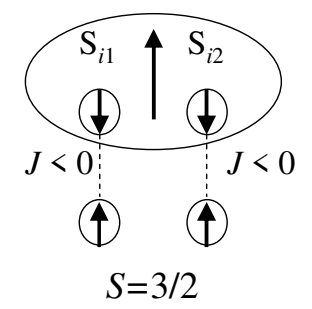

FIG. 14: Schematic picture of intramolecular spin alignment of doped system. In the case of $J>0$ : (a) $S=3 / 2$ and (b) $S=1 / 2$; in the case of $J<0$ : (c) $S=1 / 2$ and (d) $S=3 / 2$.

Nanotechnology Materials Program.
1 K. Itoh: Chem. Phys. Lett. 1 (1967) 235.

2 E. Wasserman, R. W. Murray, W. A. Yager, A. M. Trozzolo and G. Smolinsky: J. Am. Chem. Soc. 89 (1967) 5076.

3 A. Rajca: Chem. Rev. 94 (1994) 871.

4 J. S. Miller and A. J. Epstein: Angew. Chem. Int. Ed. Engl. 33 (1994) 385.

5 Molecular Magnetism: New Magnetic Materials, ed. K. Itoh and M. Kinoshita, (Kodansha Scientific, Tokyo, 2000).

6 Magnetism: Molecules to Materials, ed. J. S. Miller and M. Drillon, (Wiley-Vch, Weinheim 2001), Vol.I-III.

7 A. Izuoka, M. Hiraishi, T. Abe, T. Sugawara, K. Sato and T. Takui: J. Am. Chem. Soc. 122 (2000) 3234.

${ }^{8}$ H. Sakurai, A. Izuoka and T. Sugawara: J. Am. Chem.
Soc. 122 (2000) 9723.

9 J. Nakazaki, I. Chung, M. Matsushita, T. Sugawara, R. Watanabe, A. Izuoka and Y. Kawada: J. Mater. Chem. 13 (2003) 1011.

10 Y. Teki, S. Miyamoto, K. Iimura, M. Nakatsuji and Y. Miura: J. Am. Chem. Soc. 122 (2000) 984.

11 Y. Teki, S. Miyamoto, M. Nakatsuji and Y. Miura: J. Am. Chem. Soc. 123 (2001) 294.

12 Y. Teki, M. Nakatsuji and Y. Miura: Mol. Phys. 100 (2002) 1385.

13 Y. Teki: Polyhedron 20 (2001) 1163.

${ }^{14}$ K. Matsuda and M. Irie: J. Am. Chem. Soc. 122 (2000) 7195 . 
${ }^{15}$ K. Matsuda and M. Irie: J. Am. Chem. Soc. 122 (2000) 8309.

16 M. Matsushita, T. Nakamura, T. Momose, T. Shida, Y. Teki, T. Takui, T. Kinoshita and K. Itoh: J. Am. Chem. Soc. 114 (1992) 7470.

17 O. Sato, T. Iyoda, A. Fujishima and K. Hashimoto: Science 272 (1996) 704.

18 T. Kawamoto, Y. Asai and S. Abe: Phys. Rev. Lett. 86 (2001) 348.

19 N. Mataga: Theor. Chim. Acta 10 (1968) 372.

20 A. A. Ovchinnikov: Theor. Chim. Acta 47 (1978) 297.

21 N. Tyutyulkov, P. Schuster and O. Polansky: Theor. Chim. Acta 63 (1983) 291.

${ }^{22}$ K. Nasu: Phys. Rev. B 33 (1986) 330.

${ }^{23}$ H. Fukutome, A. Takahashi and M. Ozaki: Chem. Phys. Lett. 133 (1987) 34.

24 B. Sinha, I. D. L. Albert and S. Ramasesha: Phys.Rev. B 42 (1990) 9088.

25 Z. Fang, Z. L. Liu and K. L. Yao: Phys. Rev. B 49 (1994) 3916.

${ }^{26}$ K. Yoshizawa, K. Tanaka and T. Yamabe: J. Phys. Chem. 98 (1994) 1851.

27 S. Yamanaka, T. Kawakami, M. Okumura and K. Yamaguchi: Chem. Phys. Lett. 233 (1995) 257.

${ }^{28}$ H. Mizouchi, A. Ikawa and H. Fukutome: J. Phys. Soc.
Jpn. 66 (1997) 881

29 Y. Chen and H. Chen: J. Phys.: Condens. Matter 13 (2001) 10057.

30 R. Arita, Y. Suwa, K. Kuroki and H. Aoki: Phys. Rev. Lett. 88 (2002) 127202.

31 E. H. Lieb: Phys. Rev. Lett. 62 (1989) 1201.

32 Y. Nagaoka: Phys. Rev. 147 (1966) 392.

33 W. P. Su, J. R. Schrieffer and A. J. Heeger: Phys. Rev. B 22 (1980) 2099.

34 P. Huai, Y. Shimoi and S. Abe: Phys. Rev. Lett. 90 (2003) 207203.

35 P. Huai, Y. Shimoi and S. Abe: Synth. Met. 137 (2003) 1255.

36 H. Nishide, N. Yoshioka, K. Inagaki and E. Tsuchida: Macromolecules 21 (1988) 3119.

37 H. Iwamura and R. D. McKelvey: Macromolecules 21 (1988) 3386.

38 E. Jeckelmann and D. Baeriswyl: Synth. Met. 65 (1994) 211.

39 E. Lieb and D. Mattis: J. Math. Phys. 3 (1962) 749.

40 M. Kuwabara, Y. Shimoi and S. Abe: J. Phys. Soc. Jpn. 67 (1998) 1521.

41 See preliminary results on fused carbon rings in: P. Huai, Y. Shimoi and S. Abe: Curr. Appl. Phys. 4 (2004) 539. 